\title{
Evaluating Daratumumab in the Treatment of Multiple Myeloma: Safety, Efficacy and Place in Therapy
}

This article was published in the following Dove Press journal: Cancer Management and Research

\author{
Danai Dima' \\ Joshua Dower' \\ Raymond L Comenzo ${ }^{2}$ \\ Cindy Varga ${ }^{2}$ \\ 'Department of Medicine, Tufts Medical \\ Center, Boston, MA 02III, USA; ${ }^{2}$ The \\ John Conant Davis Myeloma and Amyloid \\ Program, Division of Hematology- \\ Oncology, Tufts Medical Center, Boston, \\ MA 02III, USA
}

\begin{abstract}
Despite the tremendous advances in the treatment of multiple myeloma, mortality remains significant, highlighting the need for new effective strategies. In recent years, daratumumab, a novel human monoclonal antibody, binding CD38, has dramatically improved outcomes either as monotherapy or in combination with traditional regimens. Originally approved for relapsed/refractory multiple myeloma, this breakthrough medication is now being used as frontline therapy in patients with newly diagnosed multiple myeloma regardless of transplant eligibility, with trials showing promising results. Its tolerable sideeffect profile and enhanced efficacy have led to its widespread incorporation into the management of multiple myeloma and further exploration about its use in other entities such as smoldering myeloma, MGUS, MGRS and amyloidosis. This comprehensive review will discuss daratumumab's mechanism of action and safety profile, as well as research which has defined its current approved indications, and ongoing clinical investigation that will define its future.
\end{abstract}

Keywords: daratumumab, newly diagnosed multiple myeloma, relapsed/refractory multiple myeloma

\section{Introduction}

Multiple myeloma (MM) is an incurable hematologic malignancy caused by the accelerated clonal proliferation of plasma cells. Generally, the abnormal plasma cell population is localized in the bone marrow with only $1-7 \%$ of patients having extramedullary disease at the time of initial diagnosis, and up to $8 \%$ of patients developing extramedullary disease at some point later in their course. ${ }^{1,2}$ The rapid growth of clonal plasma cells can lead to generalized organ dysfunction through various mechanisms. The most common presenting complications include renal failure, anemia, hypercalcemia and lytic bone lesions, the so-called "CRAB criteria."3 $\mathrm{MM}$ accounts for $1.8 \%$ of all new cancer cases and approximately $10-18 \%$ of all hematologic neoplastic processes in the US. It is more common in males than females, and in African Americans compared to other ethnicities. It is most frequently diagnosed among older individuals aged 65-74 with a 5-year survival rate of only $52.2 \%$, despite the tremendous advances and continuous evolving therapeutic strategies. ${ }^{4}$

Over the past decade, rigorous research has led to the discovery of new and innovative therapies that have been established as standard-of-care regimens in
Correspondence: Raymond L Comenzo Email rcomenzo@tuftsmedicalcenter.org

Cancer Management and Research 2020:12 789I-7903 
patients with both untreated and relapsed/resistant disease. These novel agents include proteasome inhibitors (PI; Bortezomib, Carfilzomib and Ixazomib) and immunomodulator IMiD (Thalidomide, Lenalidomide, Pomalidomide), which can be used as monotherapy or in various doublet and triplet combinations such as CyBorD and VRd. ${ }^{5-9}$ Despite the achievement of deeper remissions and improved survival with these breakthrough agents, the long-term outcomes for relapsed/refractory MM (RRMM) patients still remain poor, emphasizing the need for new effective therapies.

Monoclonal antibodies have proven to be exceptionally efficacious in several solid and hematologic malignancies. ${ }^{10}$ Development of monoclonal antibodies against promising molecular targets for MM began to take form in the 1990s. By 2008, daratumumab, a fully human monoclonal G1-k antibody against CD38 epitope on the surface of plasma cells was introduced into human studies as a Phase I clinical trial. ${ }^{11}$ Its favorable effects quickly became evident, launching further clinical trials combining daratumumab with other existing antiplasma cell therapies in both the treatment-naive and relapsed populations. In November 2015, daratumumab was approved for the treatment of RRMM patients by the US Food and Drug Administration (FDA), followed by the European Medicines Agency in May 2016. Its incorporation into clinical practice has radically shifted the treatment paradigm of $\mathrm{MM}$ and has dramatically improved outcomes by prolonging progression-free survival (PFS) and in some cases, overall survival (OS) with a relatively safe toxicity profile. In this review article, we will discuss how daratumumab has earned its rightful place in current $\mathrm{MM}$ therapy based on promising data from recent clinical trials and will explore its future role in the treatment of this complex disease.

\section{Mechanism of Action}

CD38 is a transmembrane glycoprotein with ectoenzymatic functions located predominately at the surface of plasma cells, making it a good target for novel therapeutic strategies. ${ }^{12}$ CD38 is expressed on all clonal plasma cells regardless of the spectrum of disease (ex. MGUS, SMM, active MM). As a multifunctional ectoenzyme, CD38 modulates the NAD+ catabolism and plays an important role in the synthesis of cyclic-ADP-ribose, functions that contribute to cell survival via intracellular calcium mobilization and homeostasis. Recent studies suggest that CD38 may play a role in the synthesis of extracellular adenosine, which has been implicated in immune suppression. ${ }^{13}$
Daratumumab is a human monoclonal antibody that targets a unique epitope on the CD38 glycoprotein. Daratumumab induces cell death through various Fc-dependent immune effector mechanisms. These mechanisms include complement-dependent cytotoxicity, antibody-dependent cellular cytotoxicity, antibodydependent cellular phagocytosis and apoptosis via crosslinking. ${ }^{14-16}$ Furthermore, inhibition of the CD38 ectoenzymatic function may lead to direct apoptosis induction of neoplastic cells. Another distinct mechanism is its immunomodulatory action. Specifically, daratumumab binds to the CD38 positioned on the surface of immune suppressor cells which play an important role in immune dysfunction observed in MM. These cells include regulatory $\mathrm{T}$, regulatory B and myeloid-derived suppressor cells. Daratumumab effectively destroys these suppressive cell populations, resulting in marked increase of $\mathrm{T}$ (helper CD4 + and cytotoxic $\mathrm{CD} 8+$ ) cells in the blood and bone marrow. This increase in T cells leads to enhanced activity of the immune system against the tumor with subsequent elimination of MM cells ${ }^{17,18}$ (Figure 1).

\section{Dosing, Administration and Safely Profile}

Initial studies examining daratumumab's safety, dosing, and efficacy, as monotherapy or in combinations with other agents, only included individuals with RRMM..$^{11,19-22}$ Over time, the favorable outcomes and well-manageable side effect profile of daratumumab led to further expansion of studies to include individuals with newly diagnosed MM (NDMM) irrespective of transplant eligibility. ${ }^{23-25}$ The safety and efficacy of daratumumab as monotherapy and in combination with other backbone regimens have been extensively described. The novel CD38 monoclonal antibody appears to be well tolerated without significant toxicities or reported deaths when given alone or in combination with proteasome inhibitors or immunomodulators.

The majority of clinical trials have utilized intravenous daratumumab, which is the initial formulation approved by the FDA. Recently, the subcutaneous formulation was also approved. $^{26-29}$ The recommended dosing of the intravenous daratumumab formulation is $16 \mathrm{mg} / \mathrm{kg}$ once per week for the first 8 weeks, then biweekly for the next 16 weeks, and every four weeks thereafter. This dosing and schedule were meticulously established by pharmacokinetic analysis that was conducted in the context of pivotal phase I/II clinical trials and are fixed for all cases, whether 


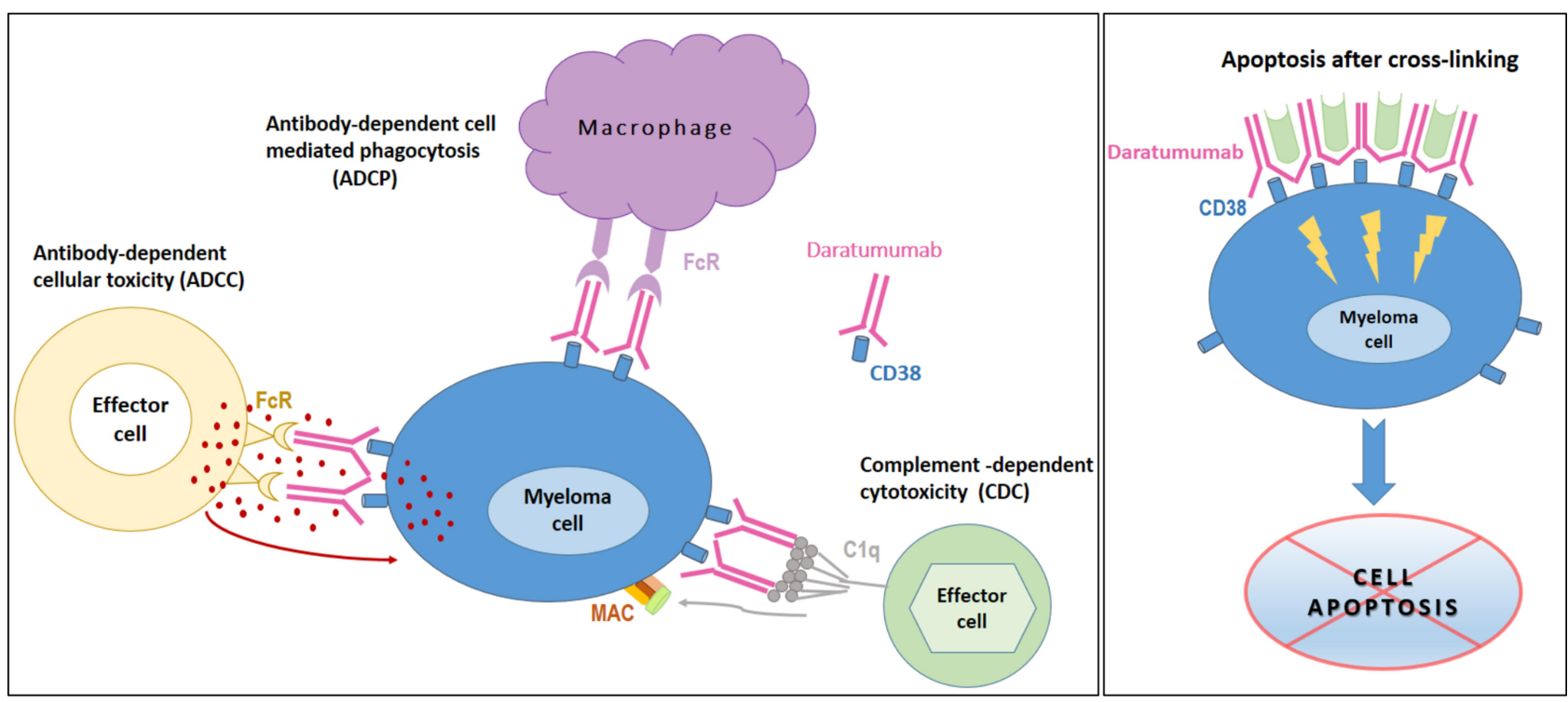

Figure I Daratumumab mechanism of action.

daratumumab is given as monotherapy or in combination with other agents and regardless of the patient or disease characteristics. $^{30,31}$ Notably, no dosing adjustments are needed in case of mild-moderate renal or hepatic impairment, although there are no current data about the pharmacokinetic profile of daratumumab in patients who are on dialysis or have severe liver failure.

The most common side effect of daratumumab when given as monotherapy or combined with any backbone regimen is infusion reactions which include headache, nasal congestion, throat irritation, cough, chills, fevers, nausea and vomiting. Severe infusion-related reactions (IRRs) include bronchospasm, dyspnea, hypoxia and hypertension. Most IRRs are mild (grade 1-2 in severity), manageable and predominantly occur during the first administration. ${ }^{11}$ Notably, the median duration of the first infusion is approximately 7 hours with subsequent infusions being shorter (approximately $1.5 \mathrm{~h}$ ). In an effort to limit the incidence and severity IRRs by reducing the infusion time, FDA approved the splitting of the first daratumumab dose over 2 consecutive days ( $8 \mathrm{mg} / \mathrm{kg}$ on days 1 and 2 of cycle 1$)$, since this strategy was proven to not alter the weekly pharmacokinetic concentrations of the novel antibody. ${ }^{32}$

The use of pre-medications approximately 1 hour prior to each daratumumab infusion also prevent the occurrence of IRRs. Pre-medications include: [1] methylprednisolone $100 \mathrm{mg}$ IV or dexamethasone $20 \mathrm{mg}$ IV for the first two infusions and methylprednisolone $60 \mathrm{mg}$ IV or dexamethasone $12 \mathrm{mg}$ (oral or IV) prior to following infusions, [2] oral antipyretics (acetaminophen 650-1000 mg orally), and (iii) antihistamine (diphenhydramine 25 to $50 \mathrm{mg}$ orally or IV). There is encouraging evidence about the use of Montelukast (leukotriene receptor antagonist) as a pre-medication agent. One study with 60 individuals concluded that the addition of Montelukast more than $30 \mathrm{~min}$ prior to the first daratumumab infusion reduced the rate of IRRs by one-third. ${ }^{33}$

Nearly all reactions occur during daratumumab administration or within 4 hours of completing infusion; rarely reactions happen up to 48 hours after infusion. In order to avoid delayed reactions, the patients are post-medicated with oral corticosteroids for 2 days following daratumumab. For individuals with underlying lung pathology including asthma and/or chronic obstructive pulmonary disease, postmedications should also include diphenhydramine $25-50 \mathrm{mg}$ oral or equivalent on the 2 days following daratumumab infusions and inhaled short-acting beta- 2 adrenergic receptor agonist as well as, inhaled corticosteroids along with longacting bronchodilators in more severe cases. ${ }^{34}$

Other common side effects include infections with respiratory involvement being the most common (upper respiratory infections, pneumonia) as well as cytopenias (most commonly anemia, neutropenia thrombocytopenia) which are believed to be synergistic, due to the combination of daratumumab with traditional regimens. ${ }^{35}$

\section{Relapsed/Refractory MM}

Historically, patients with MM who had been treated with prior lines of therapy had very limited options at disease 
relapse. $^{36-38}$ Daratumumab was initially studied for its role in managing these patients and the results were promising (Table 1). In a phase I trial (GEN501), there was no maximum tolerated dose (MTD) when using daratumumab as monotherapy in patients with RRMM despite having been heavily pretreated median of 4 prior therapies, 64\% double refractory to PI and IMiD. ${ }^{11}$ Additionally, patients who received $16 \mathrm{mg} / \mathrm{kg}$ achieved an overall response rate (ORR) of $36 \%$ compared to only $10 \%$ in the lower dose groups. SIRIUS, was a Phase II trial that reported an ORR of $29.2 \%$ after treating a RRMM population (median 5 prior lines of therapy) using $16 \mathrm{mg} / \mathrm{kg}$ of daratumumab. ${ }^{19}$ The 1 -year OS was 65\%. In a two-part phase I trial (GEN503), daratumumab was combined with lenalidomide and dexamethasone. The findings were astounding with an ORR $81 \%{ }^{20}$ These studies demonstrated that daratumumab was overall well tolerated and could be cleared for further studies.

Two landmark Phase III trials studied the synergism of daratumumab when combined with bortezomib and lenalidomide in RRMM. The CASTOR trial randomly assigned 499 patients with $\geq 1$ prior treatment to receive bortezomib and dexamethasone (D-Vd) with or without daratumumab (Vd). Daratumumab significantly improved the ORR and PFS across all subgroups. The D-Vd arm had a 61\% lower risk for death or disease progression compared to the $\mathrm{Vd}$ arm. $^{25}$ Given daratumumab's unquestionable benefit the study was stopped early and patients in the $\mathrm{Vd}$ group had the option to receive daratumumab. After a median followup of 19.4 months, D-Vd arm continued to maintain significant benefit with respect to response rates and PFS. ${ }^{39}$

Similarly, the POLLUX trial followed 569 patients with RRMM, this time assigned to receive either lenalidomide and dexamethasone alone $(\mathrm{Rd})$ or with daratumumab (D-Rd) dosed at $16 \mathrm{mg} / \mathrm{kg}$. The 12-month PFS in the intervention arm was an amazing $83.2 \%$ compared to $60.1 \%$ in the control group, again a statistically significant difference. The ORR rate of the D-Rd group was $92.9 \%$ versus $76.4 \%$ in the $\mathrm{Rd}$ group, and the proportion of those achieving a CR or better was $43.1 \%$ vs $19.2 \%$, respectively. ${ }^{22}$ After a median follow-up of 44.3 months, daratumumab continued to offer a PFS benefit, greater overall responses as well as sustained minimal residual disease (MRD) negativity. ${ }^{40}$

In both trials, addition of daratumumab led to significantly prolonged PFS and improved responses in subgroups of patients with high and standard cytogenetic risk. Specifically, for the high-risk subgroup, CASTOR revealed that the median PFS with D-Vd was 11.2 months vs 7.2 months with $\mathrm{Vd}$. The benefit of adding daratumumab although statistically significant was less pronounced

Table I Pilot Phase Clinical Trials Evaluating Daratumumab in Patients with RRMM

\begin{tabular}{|c|c|c|c|c|c|}
\hline Study Name & Phase & $\mathbf{N}$ & $\begin{array}{l}\text { Number of Prior } \\
\text { Treatments }\end{array}$ & Regimen & Results \\
\hline GEN50I & $|-| \mid$ & 104 & 4 & $\begin{array}{l}\text { Dara monotherapy } \\
\text { Dose escalation: } 0.005-24 \mathrm{mg} / \mathrm{kg} \\
\text { Expansion phase: } 8 \mathrm{mg} / \mathrm{kg} \text { and } 16 \mathrm{mg} / \\
\mathrm{kg}\end{array}$ & $\begin{array}{l}\text { No MTD } \\
\text { At } 16 \mathrm{mg} / \mathrm{kg} \text { dose: } \\
\text { - ORR } 36 \% \text { ( } \geqslant P R) \\
\text { - } 12 \text {-month OS: } 77 \% \\
\text { - mPFS } 5.6 \text { months } \\
\cdot 65 \% \text { no progression at } 12 \text { months }\end{array}$ \\
\hline SIRIUS & II & 106 & 5 & $\begin{array}{l}\text { Dara monotherapy } \\
(16 \mathrm{mg} / \mathrm{kg})\end{array}$ & $\begin{array}{l}\text { - ORR } 29.2 \% \\
\text { - I2-month OS: } 64.8 \% \\
\text { - mPFS: } 3.7 \text { month }\end{array}$ \\
\hline CASTOR & III & 499 & 2 & $\begin{array}{l}\text { Arm I: Dara-Vd } \\
\text { Arm 2: Vd (control) }\end{array}$ & $\begin{array}{l}\text { - } \downarrow \text { risk of progression by } 61 \% \\
\text { - ORR ( } \geqslant \text { VGPR) } 83.8 \% \text { vs } 63.2 \% \\
\text { - MRD negativity: II.6\% vs } 2.4 \% \\
\text { - mPFS } 16.7 \text { vs } 7.1 \text { months }\end{array}$ \\
\hline POLLUX & III & 569 & I & $\begin{array}{l}\text { Arm I: Dara-Rd } \\
\text { Arm 2: Rd (control) }\end{array}$ & $\begin{array}{l}\text { - ORR ( } \geqslant \text { VGPR) } 92.9 \text { vs } 76.4 \% \\
\text { - MRD negativity } 30.4 \% \text { vs } 5.3 \% \\
\text { - mPFS } 44.5 \text { vs } 17.5 \text { months } \\
\text { OS: NR for either group }\end{array}$ \\
\hline
\end{tabular}

Abbreviations: ORR, overall response rate; CR, complete response; VGPR, very good partial response; PR, partial response; Vd, Velcade-dexamethasone, Rd, Revlimiddexamethasone; MRD, minimal residual disease, mPFS, median progression-free survival; OS, overall survival; MTD, maximum tolerated dose. 
compared to the benefit of the standard risk subgroup. ORR was higher with $\mathrm{D}-\mathrm{Vd}$ by $81.8 \%$ versus $61.7 \%$. Similarly, in POLLUX, PFS was significantly longer in high-risk patients receiving daratumumab versus $\mathrm{Rd}$ alone (median: 26.8 vs 8.3 months). D-Rd also prolonged PFS in first relapse and PFS2. ${ }^{41}$ Again, regardless of cytogenetic risk, the rates of ORR, VRPR and CR were higher with D-Rd than with Rd.

MRD was assessed in both studies. In CASTOR, addition of daratumumab increased MRD-negative rates at all sensitivity thresholds $\left(10^{-4}, 10^{-5}, 10^{-6}\right)$, and evaluated subgroups (regardless of prior lines of therapy and cytogenetic risk) compare to $\mathrm{Vd}$ alone. Notably, only $\mathrm{D}-\mathrm{Vd}$ but not $\mathrm{Vd}$ induced MRD negativity in high-risk patients. MRDnegative status was associated with prolonged PFS in both study arms. Among patients with MRD-positive status, D-Vd prolonged PFS versus Vd. Similarly, in POLLUX, MRD negativity $\left(10^{-5}\right)$ in the D-Rd arm was significantly higher and has also continued to deepen over time compared to Rd alone. PFS was superior, but not significantly, with D-Rd versus $R d$ in patients who achieved MRD negativity and significantly prolonged with $\mathrm{D}$-Rd versus $\mathrm{Rd}$ in patients with MRD-positive status. ${ }^{22,42}$

A recent subgroup analysis of both trials revealed that patients who received daratumumab in the 65-74 and the $\geq 75$ age categories had significantly prolonged PFS compared to the rest of the cohort who did not receive the novel antibody. However, in terms of ORR, the subgroup of individuals $\geqslant 75$ years who received daratumumab did not have a statistically significant benefit, although they did have significantly higher rates of $\geqslant \mathrm{CR}$ and $\geqslant \mathrm{VGPR}^{43}$ Of note, there were limited number of patients $\geqslant 75$ years of age in both trials.

Results from these studies led to the FDA approval of daratumumab in 2015 as monotherapy for RRMM patients who had received at least 3 lines of prior therapy (including PI and IMiD) or who were double refractory to an IMiD or PI. The following year, FDA approved daratumumab with Vd or Rd for RRMM patients who had received at least one prior line of therapy.

Daratumumab is also being studied in 103 individuals with RRMM in combination with pomalidomidedexamethasone in a Phase $1 \mathrm{~b}$ trial (EQUULEUS; NCT01998971). Results so far have shown an ORR of $52.2 \%$ with VGFR achieved in $28 \%$ and CR in $6 \%$ of the participants. Most of the patients were refractory to lenalidomide (89\%), bortezomib (71\%) or both $(64 \%)$. This trial led to the 2017 FDA approval of daratumumab in combination with pomalidomide and dexamethasone for the treatment of patients with MM who have received at least 2 prior therapies including lenalidomide and a PI. In a retrospective study of 34 patients done by Emory, it was found that retreatment with daratumumab-pomalidomide-dexamethasone in patients who were previously refractory to pomalidomide and/or daratumumab resulted in response. ${ }^{44}$

One arm of the innovative phase I/II STOMP trial (NCT02343042) combines daratumumab with selinexor and dexamethasone (SDd) in patients with RRMM who had received $\geqslant 3$ prior line of therapy, in an effort to determine the maximum tolerated dose as well as the safety and efficacy of his combination. Selinexor is an oral 1st class selective inhibitor of exportin-1 (XPO1), which is the major nuclear export protein for tumor suppressor proteins and oncoprotein mRNAs. This protein is overexpressed in $\mathrm{MM}$, has been associated with drug resistance and unfavorable prognosis. Gasparetto et al announced the preliminary efficacy data at the 2019 EHA meeting, then in ASCO 2020 reporting that SDd leads to deep and durable responses. ORR was $73 \%$ in patients who had not received prior daratumumab or selinexor with mPFS of 12.5 months, whereas ORR was $69 \%$ in the entire cohort. The safety profile of SDd was manageable, with the most common grade 3/4 side effects being pancytopenia. Further results are anxiously awaited. ${ }^{45}$

Currently, daratumumab is being studied in several trials in combination with other novel agents including pomalidomide, carfilzomib (a 2nd generation PI) or ixazomib for the treatment of RRMM. ${ }^{32,44,46,47}$ Other trials are investigating combinations of daratumumab with all-trans retinoic acid (NCT02751255), venetoclax (a Bcl-2 inhibitor) (NCT03314181), or checkpoint inhibitors such as atezolizumab (NCT02431208), durvolumab (NCT02807454) and nivolumab (NCT03184194, NCT01592370). Johns Hopkins has recently developed a study combining daratumumab with CPI-444, an oral, small-molecule inhibitor targeting adenosine-2A receptors (NCT04280328). Other small molecules being paired with daratumumab include NKTR-255, an IL-15 receptor agonist (NCT04136756) and INCB001158 (NCT03837509), an investigational first-inclass, novel small molecule arginase inhibitor. These groundbreaking trials highlight the impressive anti-plasma cell activity of daratumumab and its widely positive impact on the outcomes of patients with refractory/relapse MM. These results led investigators to consider other applications for daratumumab, notably in newly diagnosed patients. 


\section{Newly Diagnosed MM}

Now, a few years since its initial approval in 2015, daratumumab is being investigated for its utility in frontline therapy. Much of the data are encouraging, reaffirming daratumumab's favorable profile and unquestionable clinical benefit.

\section{Transplant-Eligible Patients}

The traditional induction/consolidation regimen for patients who are candidates for autologous stem cell transplantation (ASCT) in North America is VRd, a three-drug regimen of bortezomib, lenalidomide and dexamethasone, followed by lenalidomide maintenance therapy after ASCT. ${ }^{9,48-51}$ Numerous studies have shown the clinical efficacy of three drug regimens for the treatment of MM. ${ }^{7,52}$ Additionally, there is evidence to suggest superiority of triple therapy versus double. ${ }^{48}$ However, with MM remaining a challenging disease with a high morbidity and mortality burden on patients, researchers are continuously looking for clinical solutions to improve patient outcomes. Daratumumab, with its success amongst patients with relapsed/refractory MM, has emerged as a new frontier for patients in first-line therapy.

The 2019 CASSIOPEIA trial was the first major phase III trial that successfully showed daratumumab's benefit when combined with traditional frontline induction/consolidation therapy prior to ASCT (Table 2). The trial compared the combination of IV daratumumab with VTd (bortezomib-thalidomide-dexamethasone) versus VTd alone as induction regimen in preparation of ASCT and for consolidation therapy. The patients who achieved a PR or better were then further randomized to receive daratumumab maintenance versus observation until disease progression. Standard risk patients in the daratumumab group maintained a significantly better and deeper responses including stringent (sCR), improved MRD negativity and PFS. sCR was seen in all subgroups with the exception of

Table 2 Pilot Clinical Trials Evaluating Daratumumab in Transplant-Eligible Patients with NDMM

\begin{tabular}{|c|c|c|c|c|}
\hline Study Name & Phase & $\mathbf{N}$ & Regimen & Results \\
\hline CASSIOPEIA & III & 1085 & $\begin{array}{l}\text { I/C: Dara-VTd vs VTd } \\
\text { Patients achieving } \geqslant \text { PR: } \\
\text { Dara maintenance vs } \\
\text { observation }\end{array}$ & $\begin{array}{l}\cdot \text { sCR } 29 \% \text { vs } 20 \% \\
\cdot \geqslant C R \quad 39 \% \text { vs } 26 \% \\
\text { - MRD negativity: } 64 \% \text { vs } 44 \% \\
\text { - } 18 \text {-mo PFS: } 93 \% \text { vs } 85 \%, \text { mPFS NR } \\
\cdot \downarrow \text { risk of progression/death by } 53 \%\end{array}$ \\
\hline GRIFFIN & $\begin{array}{l}\mathrm{I}^{[1]} \\
\mathrm{II}^{[\mathrm{g}]}\end{array}$ & $\begin{array}{l}\text { I: } 16 \\
\text { II: } 224\end{array}$ & $\begin{array}{l}\text { Dara-VRd, single arm } \\
\text { I/C: Dara-VRd vs VRd } \\
\text { M: Lena/Dara vs Lenalidomide }\end{array}$ & $\begin{array}{l}\text { - IRRs } 31 \% \\
\text { - Grade } \geqslant 3 \text { : cytopenias, febrile neutropenia, pneumonias. All } \\
\text { rare. } \\
\text { - } \geqslant \text { VGPR } 100 \%, \geqslant C R 63 \% \\
\text { - MDR negativity } 50 \% \\
\text { Preliminary data: } \\
\text { - sCR: } 50 \% \text { vs } 37 \% \\
\text { - } \geqslant \text { VGPR: } 91 \% \text { vs } 73 \% \\
\text { - MRD negativity: } 59 \% \text { vs } 24 \% \\
\text { of those achieving } \geqslant C R \\
\text { - PFS and OS immature }\end{array}$ \\
\hline PERSEUS & III & 690 & $\begin{array}{l}\text { I/C: SC Dara-VRd vs VRd } \\
\text { M: Lena/SC Dara vs Lena }\end{array}$ & Data not yet mature \\
\hline $\begin{array}{l}\text { EMN I8 } \\
\text { (NCT0389673) }\end{array}$ & II & 400 & $\begin{array}{l}\text { I/C: Dara-VRd vs VRd } \\
\text { Patients achieved } \geqslant \text { PR: } \\
\text { M: Ixazomib/Dara vs Ixazomib }\end{array}$ & Data not yet mature \\
\hline $\begin{array}{l}\text { MUKnineb } \\
\text { (NCT03I88I72) }\end{array}$ & II & $\begin{array}{l}95 \\
\text { Single arm } \\
\text { High-risk } \\
\text { patients }\end{array}$ & $\begin{array}{l}\text { I: Dara-VRd + low dose } \\
\text { Cytoxan } \\
\text { C: Dara-VRd, Dara-VR } \\
\text { M: Dara/Lena }\end{array}$ & Data not yet mature \\
\hline
\end{tabular}

Abbreviations: I/C, induction/consolidation; M, maintenance; NR, not reached; SC, subcutaneous; PFS, progression-free survival; OS, overall survival; sCR, stringent complete response; CR, complete response; VGPR, very good partial response; PR, partial response; OS, overall survival; MRD, minimal residual disease; IRR, infusionrelated reactions; VRd, Velcade-Revlimid-dexamethasone; VTd, Velcade-thalidomide-dexamethasone. 
patients with high-risk cytogenetics and ISS stage III disease. The overall serious adverse events (AEs) were similar in both arms. This groundbreaking study led to FDA approval for daratumumab (9/2019) to be used as induction and consolidation for transplant-eligible NDMM patients. $^{53}$

Several other trials are currently ongoing to further investigate how daratumumab can be used in transplanteligible candidates. The GRIFFIN phase I/II trial (NCT02874742) is examining the CR rates of 224 patients divided into two groups randomly assigned to receive either D-VRd or VRd as induction/consolidation, followed by lenalidomide/daratumumab vs lenalidomide maintenance, respectively. Preliminary results are reporting that daratumumab improved sCR rates except in patients with high-risk disease. Among patients who had achieved a CR, the daratumumab group reported higher MRD negativity ${ }^{54}$ (Table 2). The PERSEUS (NCT03710603) phase III trial takes this further and will look at 690 patients in the same treatment arms, hoping to see an improved PFS rate of the daratumumab group vs the control group (Table 2). ${ }^{55}$ Interestedly, this study used the subcutaneous formulation of daratumumab instead of the traditional IV, in an effort to limit side effects and infusion times. As outlined in Table 2, there are numerous other subsets of studies examining how daratumumab may play a role alone or in concert with other therapies prior to ASCT.

Daratumumab is also being also been studied as maintenance post ASCT. DRAMMATIC (NCT04071457) phase III study compares daratumumab and lenalidomide versus Lenalidomide alone as maintenance therapy post ASCT, followed by MRD assessment and continuation of therapy based on MRD status. Similarly, AURIGA (NCT03901963) phase III trial compares the combination of SC daratumumab + lenalidomide to lenalidomide maintenance in MRD-positive patients after ASCT. Another smaller phase II single-arm clinical trial in the City of Hope Medical Center studies the effectiveness of daratumumab monotherapy as consolidation/maintenance (NCT03346135), with primary outcome being the PFS.

Some of the data emerging from these studies are encouraging and suggest that adding daratumumab to traditional triplet regimens for pre-transplant induction, consolidation and subsequent maintenance therapy has favorable outcomes in achieving better, faster and deeper responses. So far, preliminary data from the above phase III studies suggest that standard risk patients are the ones who benefit the most from daratumumab therapy.
However, daratumumab's impact will not be completely understood until these studies are completed. Interestingly, a meta-analysis of the randomized major phase III clinical trials recently presented at ASCO 2020 suggests improved PFS among patients with high-risk cytogenetics, though final conclusions cannot be made until these studies are finalized. $^{56}$ For now, using Daratumumab as upfront induction and consolidation in ASCT eligible candidates with NDMM is considered by clinicians for select patients with a heavy burden of disease who have had suboptimal response to conventional induction treatments. This may change as more information becomes available.

\section{ASCT Ineligible Patients}

Patients with NDMM who are ineligible for ASCT carry their own challenges notably age and co-morbidities. ${ }^{58} \mathrm{Rd}$ (lenalidomide and dexamethasone), with other treatment regimens building upon this double as tolerated, is often strategy in fragile patients. ${ }^{59-61}$ Reduced-dose triplet regimens such as RVD-lite have been developed for transplant-ineligible patients. ${ }^{62}$ Just as with ASCT eligible candidates, there are several drug combinations using daratumumab which are currently being evaluated for use in transplant-ineligible patients. Results of these trials should have significant implications in the treatment of this subset of patients in the near future (Table 3).

The key phase III MAIA (NCT02252172) trial investigated the benefit of adding daratumumab to the standard $\mathrm{Rd}$ as frontline treatment. Participants were randomly assigned to receive D-Rd vs Rd. This study included 737 patients, $42 \%$ of whom were older than 75 years old, raising the external validity of the study as its population is relatively representative of the true myeloma population. Preliminary results are reported that patients in the D-Rd arm sustain better responses, as evident by the by higher rates of MRD negativity and the significantly better ORR. The PFS benefit was improved in the daratumumab arm as well, though not sustained in the subgroup of patients with high-risk cytogenetics. Risk for disease progression or death was double in the $\mathrm{Rd}$ group. ${ }^{63}$ This trial led to the 2019 FDA approval of D-Rd for initial treatment of newly NDMM patients who are ineligible for $\mathrm{ASCT}^{64}$. In realworld practice, D-Rd can be strongly considered as a reasonable alternative in cases of potentially increased toxicity with the well-established standard-of-care bortezomib-based regimens.

The pivotal ALCYONE (NCT02195479) trial is currently ongoing, investigating the potential benefit of four vs 
Table 3 Pilot Phase III Trials Evaluating Daratumumab in Transplant-Ineligible Patients with NDMM

\begin{tabular}{|c|c|c|c|c|}
\hline Study Name & Phase & $\mathbf{N}$ & Regimen & Results \\
\hline MAIA & III & 737 & $\begin{array}{l}\text { Arm I: IV Dara-Rd } \\
\text { Arm 2: Rd }\end{array}$ & $\begin{array}{l}\cdot \geqslant \text { CR: } 47.6 \% \text { vs } 24.9 \% \\
\text { - } \geqslant \text { VGFR: } 79.3 \% \text { vs } 53.1 \% \\
\text { - } 30 \text {-mo-PFS: } 70.6 \% \text { vs } 55.6 \% \\
\text { - mPFS: NR vs } 31.9 \text { months } \\
\text { - MRD negativity: } 24.2 \% \\
\text { vs. } 7.3 \%\end{array}$ \\
\hline ALCYONE & III & 706 & $\begin{array}{l}\text { Arm I: Dara-VMP } \\
\text { Arm 2: VMP }\end{array}$ & $\begin{array}{l}\text { - ORR: } 90.9 \% \text { vs } 73.9 \% \\
\text { - } \geqslant \text { CR: } 46 \% \text { vs } 25 \% \\
\text { - } \geqslant \text { VGPR: } 73 \% \text { vs } 50 \% \\
\text { - } 36 \text {-mo OS rate: } 78 \% \text { vs } \\
\text { 67.9\%; mOS NR } \\
\text { - mPFS: } 36.4 \text { vs } 19.3 \text { months, } \\
\text { - MRD negativity: } 28 \% \text { vs } 7 \%\end{array}$ \\
\hline CEPHEUS & III & 395 & $\begin{array}{l}\text { Arm I: VRd, then mRd } \\
\text { Arm 2: SC Dara-VRd, then mDara-Rd }\end{array}$ & Data not mature yet \\
\hline GEM20I7FIT & III & 300 & $\begin{array}{l}\text { Arm I: VMP, then Rd } \\
\text { Arm 2: KRd } \\
\text { Arm 3: Dara- KRd }\end{array}$ & Data not mature yet \\
\hline $\begin{array}{l}\text { LYRA }^{69} \\
(\text { NCT0295I819) }\end{array}$ & II & $\begin{array}{l}\text { I0I } \\
87 \text { NDMM ASCT } \\
\text { ineligible, I4 RRMM }\end{array}$ & $\begin{array}{l}\text { Single Arm: } \\
\text { D-VCd induction, then ASCT/consolidation (only } \\
\text { eligible patients), then mDara }\end{array}$ & $\begin{array}{l}\text { NDMM patients: } \\
\text { - CR+VGPR } 55.8 \% \text { at the } \\
\text { end of induction } \\
\text { - mTime } \geqslant \text { PR: } 10 \text { months } \\
\text { - mTime } \geqslant \text { VGPR: } 46 \text { months, } \\
\text { - I2-month PFS: } 87.9 \% \text {, } \\
\text { mPFS: NR } \\
\text { - I2-month OS: } 98.8 \%\end{array}$ \\
\hline
\end{tabular}

Abbreviations: $m$, maintenance; NR, not reached; SC, subcutaneous; mPFS, median progression-free survival; OS, overall survival; CR, complete response; VGPR, very good partial response; OS, overall survival; MRD, minimal residual disease; VCd, Velcade-cyclophosphamide-dexamethasone; VTd, Velcade-thalidomide-dexamethasone; VMP, Velcade-melphalan-prednisone; KRd, Kyprolis-Revlimid-dexamethasone.

three drug regimens in patients who are ineligible for transplant. Here, patients were randomized to receive VMP, with or without daratumumab. The D-VMP arm has thus far shown a significantly longer PFS which, over time, translated into an OS benefit, improvement in hematologic response and sustained MRD negativity. ${ }^{65,66}$ PFS benefit was sustained for patients $>75$ years and those with poor prognosis (ISS stage III, renal failure, high-risk cytogenetics). OS benefit across all subgroups, but less pronounced in patients with high-risk cytogenetic profile. Most of the side effects were evenly balanced between the 2 arms, except for infections which were more frequent and severe in the daratumumab group; the most common grade 3/4 infection was pneumonia. Subsequently, the FDA has also approved the D-VMP combination in May 2018 for initial treatment of NDMM patients who are ineligible for ASCT. ${ }^{64}$ It should be noted that patients in both arms received subsequent therapy upon relapse, and so it is unclear whether the OS benefit was due to daratumumab or subsequent therapy.

Other ongoing phase III trials are underway to evaluate daratumumab's role for treatment of NDMM patients who cannot receive transplant. CEPHEUS (NCT03652064) is evaluating the combination daratumumab + VRd followed by dara-Rd maintenance. ${ }^{67}$ GEM2017FIT (NCT03742297), is comparing three combinations: VMP followed by $\mathrm{Rd}$, vs carfilzomib-Rd, vs carfilzomib-Rd plus daratumumab in fit elderly individuals between 65 and 80 years of age. Results of these studies are pending.

Most recently there are several ongoing studies investigating the combination of daratumumab with novel treatments such as the 2nd generation PIs (carfilzomib or ixazomib) as frontline for both transplant eligible and ineligible patients with NDMM. The most frequent combinations tested are daratumumab with 
carfilzomib-lenalidomide-dexamethasone or daratumumab with ixazomib-lenalidomide-dexamethasone. ${ }^{68}$

\section{Interference with SPE/IFE}

According to the IMWG criteria, treatment response assessment in $\mathrm{MM}$ is performed by periodic monitoring of the monoclonal paraprotein with serum protein electrophoresis (SPE) and immunofixation (IFE). Daratumumab is an IgG kappa monoclonal antibody, and thus may be detected on SPEP and/or IFE, similarly to endogenous monoclonal immunoglobulin, leading to false-positive results. ${ }^{70,71}$ This can be particularly problematic in cases of IgG kappa disease treated with daratumumab, since a positive assay result may represent daratumumab itself rather than the presence of an abnormal paraprotein. This often has negative impact on initial evaluation of complete responses and leads to an increased frequency of VGPR with underrepresentation of CR. It is highly likely that several of the above trials overreported VGPRs that might actually be CRs. Therefore, it is possible that daratumumab has a greater efficacy than is being reported in the above studies. A recently developed daratumumab-specific IFE assay (DIRA) with an antidaratumumab antibody has helped to overcome this interference and accurately classify hematologic response. ${ }^{72}$ Molecular mass spectrometry mechanisms have also been developed and are slowly being utilized to help differentiate between monoclonal antibodies and abnormal proteins, reducing the frequency of this phenomenon. ${ }^{73-75}$

\section{Blood Compatibility}

Another challenge with daratumumab use comes from its ability to bind to CD38 on red blood cells. This interaction may result in pan-agglutination in vitro leading to panreactivity in indirect antiglobulin tests, antibody detection tests, antibody identification panels, and anti-human globulin crossmatches. However, the drug does not affect the patient's $\mathrm{ABO}$ blood typing and immediate spin crossmatch. In the clinical setting, this in vitro interference can translate to unanticipated delays in blood transfusions, raising safety concerns. One successful method to overcome this issue is dithiothreitol (DTT) treatment of RBC screening cells which eliminates the interference with daratumumab. DTT disrupts the extracellular bonds of CD38 and prevents daratumumab binding. ${ }^{76}$ Another approach is, neutralization of daratumumab with recombinant human anti-daratumumab idiotype antibodies, but its use is still limited. An easy strategy is to perform a baseline type and screen and phenotype prior to starting daratumumab therapy. ${ }^{77}$ These patients should carry transfusion identification cards throughout their treatment with the novel antibody and up to 6 months after therapy completion. In emergency situations, blood banks should release non-crossed matched $\mathrm{ABO}$ and $\mathrm{Rh}$ compatible red cells.

\section{Future Role and Applications SC Daratumumab}

As daratumumab becomes a well-established medication in the treatment of multiple myeloma, investigators have begun to look into novel applications to utilize this medication further. A number of clinical trials are currently underway looking into the safety, efficacy and utility of SC administration of daratumumab. Additionally, these studies are investigating whether subcutaneous administration of daratumumab decreases infusion reactions and infusion times, improving patient tolerance of the agent. PAVO (NCT02519452) phase 1b was the first trial that confirmed the safety of SC daratumumab as monotherapy. ${ }^{26-28}$ A subsequent Chinese phase I clinical study is following 20 participants over 2 years to determine the rate of adverse effects in relation to the concentration of the drug at the beginning of each 28-day cycle. The phase III international trial COLUMBA (NCT03277105) with 522 participants is taking this further, comparing the ORR of SC daratumumab vs IV daratumumab. Interim data suggest that dara-SC is non-inferior to dara-IV and is associated with a lower rate of infusion reaction rate. ${ }^{78}$ Additionally, the infusion time was decreased from hours to minutes.

Besides establishing the efficacy of monotherapy SC daratumumab monotherapy, there are other ongoing trials examining the efficacy of SC daratumumab in combination with other novel agents PLEIADES (NCT03412565) is a phase II trial that is currently adding SC daratumumab to various standard regimens (VRd, VMP, Rd, and $\mathrm{Kd}$ ) ${ }^{79}$ So far results reveal that SC daratumumab leads to similar efficacy as the IV formulation. Outcomes here will compare the treatment arms to phase III data on the established agents without daratumumab to again see how these novel regimens will help patients achieve a PR or better.

\section{Smoldering MM (SMM)}

There is also a move to consider the use of daratumumab in the treatment of monoclonal gammopathy of unknown significance (MGUS) and smoldering MM. The CENTAURUS (NCT02316106) phase II trial is evaluating 3 different daratumumab schedules/doses in an effort to 
determine whether daratumumab monotherapy can delay progression to overt $\mathrm{MM}$. Interim results suggest that daratumumab does have single-agent activity in this patient population; however, the primary endpoints of $\mathrm{CR}>15 \%$ and median PFS $\geqslant 24$ months were not met. ${ }^{80}$ A phase II trial from Dana Farber is currently evaluating the efficacy of daratumumab for the treatment of high-risk MGUS or low-risk smoldering MM, following patients for 2 years to see how many will achieve VPGR or better. The active phase III AQUILA (NCT03301220) trial is randomly assigning patients with smoldering $\mathrm{MM}$ to either an active monitoring arm without intervention or an experimental arm in which they receive SC daratumumab for 39 cycles or 36 weeks. ${ }^{81}$ The primary outcome measure here is PFS, measured as time to development of active multiple myeloma, with all patients being screened for this transition every 12 weeks. Another ongoing phase III clinical trial (NCT03937635) with 288 participants is currently evaluating how daratumumab can be utilized with lenalidomide/dexamethasone in the treatment of highrisk Smoldering MM, this time looking at the OS between the two arms. Overall, while there is hope that daratumumab may be of value in the treatment of Smoldering MM, it is crucial to scrutinize its toxicity profile in this particular patient population, since the potential adverse effects of this agent may lead to increased morbidity and mortality relative to electing a periodic monitoring approach.

\section{Conclusion}

While daratumumab is a relatively new agent in the treatment of MM, studies continue to show the promise this medication brings to an incurable disease. We have already seen its efficacy in the treatment of RRMM and are now starting to use this agent for management of NDMM. Additionally, ongoing clinical trials are beginning to show decreased infusion reactions and infusion times when utilizing subcutaneous daratumumab versus the traditional intravenous formulation.

With all these positive results, it will be interesting to see other potential uses for this medication. The authors of this review have used daratumumab urgently as monotherapy in patients hospitalized with cast nephropathy and seen rapid improvements in renal function due to the medication's ability to reduce serum light chains rapidly, avoiding the need for plasmapheresis. Moving forward, it is possible to imagine daratumumab's role in the treatment of other plasma cell dyscrasias such as plasma cell leukemia, monoclonal gammopathy of renal significance (NCT03095118),
Waldenstrom macroglobulinemia (NCT03187262) and amyloidosis (NCT02841033, NCT03201965, NCT03283917, NCT04270175), as well as other hematologic malignancies such as plasmablastic lymphoma (NCT04139304), myelodysplastic syndrome (NCT03067571, NCT03011034), acute myeloid (NCT03537599, NCT03067571), acute lymphoblastic and chronic lymphocytic leukemia (NCT03734198, NCT04230304, NCT03447808), and possibly even in autoimmune disorders. Recently, MD Anderson is further exploring daratumumab's role in solid tumors including kidney, prostate and bladder cancer (NCT03473730, NCT03177460). Other potential uses include desensitization prior to kidney (NCT04204980) and heart (NCT04088903) transplantation as well. Further investigations into daratumumab's role in these diseases will be critical to help utilize this medication in clinical practice, develop new monoclonal antibodies, and help our patients avoid unnecessary morbidity and mortality.

\section{Abbreviation}

MM, multiple myeloma; MGUS, monoclonal gammopathy of undetermined significance; NDMM, newly diagnosed multiple myeloma; RRMM, relapsed/refractory multiple myeloma; MRD, minimal residual disease; PFS, progression-free survival; OR, overall response; sCR, stringent complete response; VGPR, very good partial response; ASCT, autologous stem cell transplant; CyBorD, cyclophosphamide bortezomib dexamethasone; IMiD, immunomodulatory drug; IRR, Infusion-related reactions; IV, intravenous; $\mathrm{Kd}$, carfilzomib dexamethasone; KRd, carfilzomib lenalidomide dexamethasone; PI, proteasome inhibitor; $\mathrm{Rd}$, lenalidomide dexamethasone; $\mathrm{Vd}$, bortezomib dexamethasone; VMP, bortezomib melphalan prednisone; VRd, bortezomib lenalidomide dexamethasone; SC, subcutaneous.

\section{Disclosure}

Dr Danai Dima and Dr Joshua Dower are co-first authors. Dr Raymond Comenzo reports a patent WO2016187546A1 issued. The authors report no other conflicts of interest in this work.

\section{References}

1. Varettoni M, Corso A, Pica G, Mangiacavalli S, Pascutto C, Lazzarino M. Incidence, presenting features and outcome of extramedullary disease in multiple myeloma: a longitudinal study on 1003 consecutive patients. Ann Oncol. 2010;21(2):325-330. doi:10.1093/ annonc/mdp329 
2. Short KD, Rajkumar SV, Larson D, et al. Incidence of extramedullary disease in patients with multiple myeloma in the era of novel therapy, and the activity of pomalidomide on extramedullary myeloma. Leukemia. 2011;25(6):906-908. doi:10.1038/leu.2011.29

3. Palumbo A, Anderson K. Multiple myeloma. $N$ Engl J Med. 2011;364(11):1046-1060. doi:10.1056/NEJMra1011442

4. Surveillance, Epidemiology, and End Results (SEER) Program. Cancer stat facts: myeloma.

5. Stege CAM, Nasserinejad K, Levin M-D, et al. Efficacy and tolerability of Ixazomib, Daratumumab and Low Dose Dexamethasone (IDd) in Unfit and Frail Newly Diagnosed Multiple Myeloma (NDMM) patients; first interim safety analysis of the Phase II HOVON 143 Study. Blood. 2018;132(Supplement 1):596. doi:10.1182/blood-2018-99-114668

6. Jakubowiak AJ, Dytfeld D, Griffith KA, et al. A phase 1/2 study of carfilzomib in combination with lenalidomide and low-dose dexamethasone as a frontline treatment for multiple myeloma. Blood. 2012;120(9):1801-1809. doi:10.1182/blood-2012-04-422683

7. Kumar S, Flinn I, Richardson PG, et al. Randomized, multicenter, Phase 2 study (EVOLUTION) of combinations of bortezomib, dexamethasone, cyclophosphamide, and lenalidomide in previously untreated multiple myeloma. Blood. 2012;119(19):4375-4382. doi:10.1182/blood-2011-11-395749

8. Reeder CB, Reece DE, Kukreti V, et al. Once- versus twice-weekly bortezomib induction therapy with CyBorD in newly diagnosed multiple myeloma. Blood. 2010;115(16):3416-3417. doi:10.1182/blood2010-02-271676

9. Richardson PG, Weller E, Lonial S, et al. Lenalidomide, bortezomib, and dexamethasone combination therapy in patients with newly diagnosed multiple myeloma. Blood. 2010;116(5):679-686. doi:10.1182/ blood-2010-02-268862

10. Lu RM, Hwang YC, Liu IJ, et al. Development of therapeutic antibodies for the treatment of diseases. J Biomed Sci. 2020;27(1):1. doi:10.1186/s12929-019-0592-z

11. Lokhorst HM, Plesner T, Laubach JP, et al. Targeting CD38 with daratumumab monotherapy in multiple myeloma. $N$ Engl $\mathrm{J}$ Med. 2015;373(13):1207-1219. doi:10.1056/NEJMoa1506348

12. van de Donk N, Richardson PG, Malavasi F. CD38 antibodies in multiple myeloma: back to the future. Blood. 2018;131(1):13-29. doi:10.1182/blood-2017-06-740944

13. Horenstein AL, Quarona V, Toscani D, et al. Adenosine generated in the bone marrow niche through a CD38-mediated pathway correlates with progression of human myeloma. Mol Med. 2016;22:694-704. doi:10.2119/molmed.2016.00198

14. Hogan KA, Chini CCS, Chini EN. The multi-faceted ecto-enzyme CD38: roles in immunomodulation, cancer, aging, and metabolic diseases. Front Immunol. 2019;10:1187. doi:10.3389/fimmu.2019.01187

15. Overdijk MB, Verploegen S, Bogels M, et al. Antibody-mediated phagocytosis contributes to the anti-tumor activity of the therapeutic antibody daratumumab in lymphoma and multiple myeloma. MAbs. 2015;7(2):311-321.

16. Overdijk MB, Jansen JH, Nederend M, et al. The therapeutic CD38 monoclonal antibody daratumumab induces programmed cell death via fcgamma receptor-mediated cross-linking. J Immunol. 2016;197 (3):807-813.

17. Krejcik J, Casneuf T, Nijhof IS, et al. Daratumumab depletes CD38+ immune regulatory cells, promotes T-cell expansion, and skews T-cell repertoire in multiple myeloma. Blood. 2016;128(3):384-394. doi:10.1182/blood-2015-12-687749

18. van de Donk N. Reprint of Immunomodulatory effects of CD38-targeting antibodies. Immunol Lett. 2019;205:71-77. doi:10. 1016/j.imlet.2019.02.002

19. Lonial S, Weiss BM, Usmani SZ, et al. Daratumumab monotherapy in patients with treatment-refractory multiple myeloma (SIRIUS): an open-label, randomised, phase 2 trial. Lancet. 2016;387(100 27):1551-1560. doi:10.1016/S0140-6736(15)01120-4
20. Plesner T, Arkenau HT, Gay F, et al. Enduring efficacy and tolerability of daratumumab in combination with lenalidomide and dexamethasone in patients with relapsed or relapsed/refractory multiple myeloma (GEN503): final results of an open-label, phase 1/2 study. Br J Haematol. 2019;186(3):e35-e9. doi:10.11 11/bjh.15879

21. Usmani SZ, Nahi H, Weiss BM, et al. Safety and efficacy of daratumumab monotherapy in patients with heavily pretreated relapsed and refractory multiple myeloma: final results from GEN501 and sirius. Blood. 2017;130(Supplement 1):3107.

22. Dimopoulos MA, Oriol A, Nahi H, et al. Daratumumab, lenalidomide, and dexamethasone for multiple myeloma. $N$ Engl J Med. 2016;375(14):1319-1331. doi:10.1056/NEJMoa1607751

23. Moreau P, Mateos M-V, Bladé J, et al. An open-label, multicenter, phase $1 \mathrm{~b}$ study of daratumumab in combination with backbone regimens in patients with multiple myeloma. Blood. 2014;124(21):176. doi:10.1182/blood.V124.21.176.176

24. O’Dwyer M, Henderson R, Naicker SD, et al. CyBorD-DARA is potent initial induction for $\mathrm{MM}$ and enhances ADCP: initial results of the 16-BCNI-001/CTRIAL-IE 16-02 study. Blood Adv. 2019;3 (12):1815-1825. doi:10.1182/bloodadvances.2019000010

25. Palumbo A, Chanan-Khan A, Weisel K, et al. Daratumumab, bortezomib, and dexamethasone for multiple myeloma. $N$ Engl J Med. 2016;375(8):754-766. doi:10.1056/NEJMoa1606038

26. Usmani SZ, Nahi H, Mateos M-V, et al. Open-label, multicenter, dose escalation phase $1 \mathrm{~b}$ study to assess the subcutaneous delivery of daratumumab in Patients (pts) with relapsed or refractory multiple myeloma (PAVO). Blood. 2016;128(22):1149. doi:10.1182/blood. V128.22.1149.1149

27. Chari A, Nahi H, Mateos M-V, et al. Subcutaneous Delivery of Daratumumab in Patients (pts) with Relapsed or Refractory Multiple Myeloma (RRMM): pavo, an open-label, multicenter, dose escalation Phase 1b study. Blood. 2017;130(Supplement 1):838.

28. Usmani SZ, Nahi H, Mateos MV, et al. Subcutaneous delivery of daratumumab in relapsed or refractory multiple myeloma. Blood. 2019;134(8):668-677. doi:10.1182/blood.2019 000667

29. Mateos M-V, Nahi H, Legiec W, et al. Efficacy and safety of the randomized, open-label, non-inferiority, Phase 3 study of subcutaneous (SC) versus intravenous (IV) daratumumab (DARA) administration in patients (pts) with relapsed or refractory multiple myeloma (RRMM): COLUMBA. J Clin Oncol. 2019;37(15 suppl):8005. doi:10.1200/JCO.2019.37.15_suppl.8005

30. Clemens PL, Yan X, Lokhorst HM, et al. Pharmacokinetics of daratumumab following intravenous infusion in relapsed or refractory multiple myeloma after prior proteasome inhibitor and immunomodulatory drug treatment. Clin Pharmacokinet. 2017;56(8):915-924. doi:10.1007/ s40262-016-0477-1

31. Xu XS, Dimopoulos MA, Sonneveld P, et al. Pharmacokinetics and exposure-response analyses of daratumumab in combination therapy regimens for patients with multiple myeloma. Adv Ther. 2018;35 (11):1859-1872. doi:10.1007/s12325-018-0815-9

32. Chari A, Martinez-Lopez J, Mateos MV, et al. Daratumumab plus carfilzomib and dexamethasone in patients with relapsed or refractory multiple myeloma. Blood. 2019;134(5):421-431. doi:10.1182/blood. 2019000722

33. Chari A, Mark TM, Krishnan A, et al. Use of montelukast to reduce infusion reactions in an early access treatment protocol of daratumumab in United States patients with relapsed or refractory multiple myeloma. Blood. 2016;128(22):2142. doi:10.1182/blood.V128.22.21 42.2142

34. Moreau P, van de Donk NW, San Miguel J, et al. Practical considerations for the use of daratumumab, a novel CD38 monoclonal antibody, in myeloma. Drugs. 2016;76(8):853-867. doi:10.1007/ s40265-016-0573-4 
35. Voorhees PM, Rodriguez C, Reeves B, et al. Efficacy and updated safety analysis of a safety run-in cohort from griffin, a Phase 2 randomized study of daratumumab (Dara), Bortezomib (V), Lenalidomide (R), and Dexamethasone (D; Dara-Vrd) Vs. Vrd in Patients (Pts) with Newly Diagnosed (ND) Multiple Myeloma (MM) Eligible for High-Dose Therapy (HDT) and Autologous Stem Cell Transplantation (ASCT). Blood. 2018;132(Supplement 1):151.

36. Dimopoulos MA, Moreau P, Palumbo A, et al. Carfilzomib and dexamethasone versus bortezomib and dexamethasone for patients with relapsed or refractory multiple myeloma (ENDEAVOR): a randomised, phase 3, open-label, multicentre study. Lancet Oncol. 2016;17(1):27-38. doi:10.1016/S1470-2045(15)00464-7

37. Stewart AK, Rajkumar SV, Dimopoulos MA, et al. Carfilzomib, lenalidomide, and dexamethasone for relapsed multiple myeloma. N Engl J Med. 2015;372(2):142-152. doi:10.1056/NEJMoa1411321

38. Siegel DS, Dimopoulos MA, Ludwig H, et al. Improvement in overall survival with carfilzomib, lenalidomide, and dexamethasone in patients with relapsed or refractory multiple myeloma. J Clin Oncol. 2018;36(8):728-734. doi:10.1200/JCO.2017.76.5032

39. Spencer A, Lentzsch S, Weisel K, et al. Daratumumab plus bortezomib and dexamethasone versus bortezomib and dexamethasone in relapsed or refractory multiple myeloma: updated analysis of CASTOR. Haematologica. 2018;103(12):2079-2087. doi:10.3324/ haematol.2018.194118

40. Bahlis NJ, Dimopoulos MA, White DJ, et al. Daratumumab plus lenalidomide and dexamethasone in relapsed/refractory multiple myeloma: extended follow-up of POLLUX, a randomized, open-label, phase 3 study. Leukemia. 2020;34(7):1875-1884. doi:10.1038/s41375-020-0711-6

41. Kaufman JL, Dimopoulos MA, Leiba M, et al. Efficacy and safety of daratumumab, lenalidomide, and dexamethasone (D-Rd) in relapsed or refractory multiple myeloma (RRMM): updated subgroup analysis of POLLUX based on cytogenetic risk. J Clin Oncol. 2019;37 (15_suppl):8038. doi:10.1200/JCO.2019.37.15_suppl.8038

42. Dimopoulos MA, San-Miguel J, Belch A, et al. Daratumumab plus lenalidomide and dexamethasone versus lenalidomide and dexamethasone in relapsed or refractory multiple myeloma: updated analysis of POLLUX. Haematologica. 2018;103(12):2088-2096. doi:10. 3324/haematol.2018.194282

43. Mateos MV, Spencer A, Nooka AK, et al. Daratumumab-based regimens are highly effective and well tolerated in relapsed or refractory multiple myeloma regardless of patient age: subgroup analysis of the phase 3 CASTOR and POLLUX studies. Haematologica. 2020;105 (2):468-477. doi:10.3324/haematol.2019.217448

44. Nooka AK, Joseph NS, Kaufman JL, et al. Clinical efficacy of daratumumab, pomalidomide, and dexamethasone in patients with relapsed or refractory myeloma: utility of re-treatment with daratumumab among refractory patients. Cancer. 2019;125(17):2991-3000. doi:10.1002/cncr.32178

45. Gasparetto C, Lentzsch S, Schiller GJ, et al. Selinexor, daratumumab, and dexamethasone in patients with relapsed/refractory multiple myeloma (MM). J Clin Oncol. 2020;38(15_suppl):8510. doi:10.1200/ JCO.2020.38.15_suppl.8510

46. Kapoor P, Gertz MA, Laplant B, et al. Phase 2 trial of daratumumab, ixazomib, lenalidomide and modified dose dexamethasone in patients with newly diagnosed multiple myeloma. Blood. 2019;134 (Supplement_1):864. doi:10.1182/blood-2019-131476

47. Chari A, Suvannasankha A, Fay JW, et al. Daratumumab plus pomalidomide and dexamethasone in relapsed and/or refractory multiple myeloma. Blood. 2017;130(8):974-981. doi:10.1182/blood-2017-05-785246

48. Durie BGM, Hoering A, Abidi MH, et al. Bortezomib with lenalidomide and dexamethasone versus lenalidomide and dexamethasone alone in patients with newly diagnosed myeloma without intent for immediate autologous stem-cell transplant (SWOG S0777): a randomised, open-label, phase 3 trial. Lancet. 2017;389 (10068):519-527. doi:10.1016/S0140-6736(16)31594-X
49. Rosinol L, Oriol A, Rios R, et al. Bortezomib, lenalidomide, and dexamethasone as induction therapy prior to autologous transplant in multiple myeloma. Blood. 2019;134(16):1337-1345. doi:10.1182/ blood.2019000241

50. Attal M, Lauwers-Cances V, Marit G, et al. Lenalidomide maintenance after stem-cell transplantation for multiple myeloma. $N$ Engl J Med. 2012;366(19):1782-1791. doi:10.1056/NEJMoa1114138

51. McCarthy PL, Owzar K, Hofmeister CC, et al. Lenalidomide after stem-cell transplantation for multiple myeloma. $N$ Engl $\mathrm{J} \mathrm{Med.}$ 2012;366(19):1770-1781. doi:10.1056/NEJMoa1114083

52. Moreau P, Hulin C, Macro M, et al. VTD is superior to VCD prior to intensive therapy in multiple myeloma: results of the prospective IFM2013-04 trial. Blood. 2016;127(21):2569-2574. doi:10.1182/ blood-2016-01-693580

53. Moreau P, Attal M, Hulin C, et al. Bortezomib, thalidomide, and dexamethasone with or without daratumumab before and after autologous stem-cell transplantation for newly diagnosed multiple myeloma (CASSIOPEIA): a randomised, open-label, phase 3 study. Lancet. 2019;394(10192):29-38. doi:10.1016/S0140-6736(19)31240-1

54. Voorhees PM, Kaufman JL, Laubach JP, et al. Daratumumab, lenalidomide, bortezomib, \& dexamethasone for transplant-eligible newly diagnosed multiple myeloma: GRIFFIN. Blood. 2020. doi:10.1182/ blood.2020005288

55. Sonneveld P, Broijl A, Gay F, et al. Bortezomib, lenalidomide, and dexamethasone (VRd) \pm daratumumab (DARA) in patients (pts) with transplant-eligible (TE) newly diagnosed multiple myeloma (NDMM): A multicenter, randomized, phase III study (PERSEUS). $J$ Clin Oncol. 2019;37(15_suppl):TPS8055-TPS. doi:10.1200/ JCO.2019.37.15_suppl.TPS8055

56. Giri S, Grimshaw A, Bal S, et al. Efficacy of daratumumab in the treatment of multiple myeloma with high-risk cytogenetics: meta-analysis of randomized phase III trials. J Clin Oncol. 2020;38 (15_suppl):8540. doi:10.1200/JCO.2020.38.15_suppl.8540

57. Sherborne AL, Shah V, Ellis S, et al. Improving Outcomes for patients with high-risk myeloma via prospective trial evidence: the myeloma UK nine optimum trial. Blood. 2017;130(Supplement 1):1767.

58. Mikhael J, Ismaila N, Cheung MC, et al. Treatment of multiple myeloma: ASCO and CCO joint clinical practice guideline. $J$ Clin Oncol. 2019;37(14):1228-1263. doi:10.1200/JCO.18.02096

59. Benboubker L, Dimopoulos MA, Dispenzieri A, et al. Lenalidomide and dexamethasone in transplant-ineligible patients with myeloma. N Engl J Med. 2014;371(10):906-917. doi:10.1056/NEJMoa1402551

60. Palumbo A, Rajkumar SV, San Miguel JF, et al. International Myeloma Working Group consensus statement for the management, treatment, and supportive care of patients with myeloma not eligible for standard autologous stem-cell transplantation. J Clin Oncol. 2014;32(6):587-600. doi:10.1200/JCO.2013.48.7934

61. Cao Y, Wan N, Liang Z, et al. Treatment outcomes in patients with newly diagnosed multiple myeloma who are ineligible for stem-cell transplantation: systematic review and network meta-analysis. Clin Lymphoma Myeloma Leuk. 2019;19(8):e478-e88. doi:10.1016/j.clml.2019.04.009

62. O'Donnell EK, Laubach JP, Yee AJ, et al. A phase 2 study of modified lenalidomide, bortezomib and dexamethasone in transplant-ineligible multiple myeloma. Br J Haematol. 2018;182 (2):222-230. doi:10.1111/bjh.15261

63. Facon T, Kumar S, Plesner T, et al. Daratumumab plus lenalidomide and dexamethasone for untreated myeloma. N Engl J Med. 2019;380 (22):2104-2115. doi:10.1056/NEJMoa1817249

64. Darzalex Approval History. 2019.

65. Mateos MV, Dimopoulos MA, Cavo M, et al. Daratumumab plus bortezomib, melphalan, and prednisone for untreated myeloma. N Engl J Med. 2018;378(6):518-528. doi:10.1056/NEJMoa1714678

66. Mateos MV, Cavo M, Blade J, et al. Overall survival with daratumumab, bortezomib, melphalan, and prednisone in newly diagnosed multiple myeloma (ALCYONE): a randomised, open-label, phase 3 trial. Lancet. 2020;395(10218):132-141. doi:10.1016/S0140-6736(19)32956-3 
67. Zweegman S, Usmani SZ, Chastain K, et al. Bortezomib, lenalidomide, and dexamethasone (VRd) \pm daratumumab (DARA) in patients (pts) with newly diagnosed multiple myeloma (NDMM) for whom transplant is not planned as initial therapy: a multicenter, randomized, phase III study (CEPHEUS). J Clin Oncol. 2019;37(15_suppl): TPS8056-TPS. doi:10.1200/JCO.2019.37.15 suppl.TPS8056

68. Chari A, Usmani SZ, Krishnan A, et al. Daratumumab (DARA) in Combination with Carfilzomib, Lenalidomide, and Dexamethasone (KRd) in Patients with Newly Diagnosed Multiple Myeloma (MMY1001): updated results from an open-label, Phase 1b study. Blood. 2017;130(Supplement 1):3110.

69. Yimer H, Melear J, Faber E, et al. Daratumumab, bortezomib, cyclophosphamide and dexamethasone in newly diagnosed and relapsed multiple myeloma: LYRA study. $B r \quad J$ Haematol. 2019;185 (3):492-502. doi:10.1111/bjh.15806

70. McCudden CR, Voorhees PM, Hainsworth SA, et al. Interference of monoclonal antibody therapies with serum protein electrophoresis tests. Clin Chem. 2010;56(12):1897-1899. doi:10.1373/clinchem.20 10.152116

71. Kumar S, Paiva B, Anderson KC, et al. International Myeloma Working Group consensus criteria for response and minimal residual disease assessment in multiple myeloma. Lancet Oncol. 2016;17(8) e328-e46. doi:10.1016/S1470-2045(16)30206-6

72. McCudden C, Axel AE, Slaets D, et al. Monitoring multiple myeloma patients treated with daratumumab: teasing out monoclonal antibody interference. Clin Chem Lab Med. 2016;54(6):1095-1104. doi:10.1515/cclm-2015-1031

73. Mills JR, Barnidge DR, Murray DL. Detecting monoclonal immunoglobulins in human serum using mass spectrometry. Methods. 2015;81:56-65. doi:10.1016/j.ymeth.2015.04.020

74. Moore LM, Cho S, Thoren KL. MALDI-TOF mass spectrometry distinguishes daratumumab from M-proteins. Clin Chim Acta. 2019;492:91-94. doi:10.1016/j.cca.2019.02.017
75. Vakili H, Koorse Germans S, Dong X, et al. Complete depletion of daratumumab interference in serum samples from plasma cell myeloma patients improves the detection of endogenous M-proteins in a preliminary study. Diagnostics (Basel). 2020;10:4.

76. Chapuy CI, Nicholson RT, Aguad MD, et al. Resolving the daratumumab interference with blood compatibility testing. Transfusion. 2015;55(6 Pt 2):1545-1554. doi:10.1111/trf.13069

77. Oostendorp M, Lammerts van Bueren JJ, Doshi P, et al. When blood transfusion medicine becomes complicated due to interference by monoclonal antibody therapy. Transfusion. 2015;55(6 Pt 2):15 $55-1562$.

78. San-Miguel J, Usmani SZ, Mateos MV, et al. Subcutaneous daratumumab in patients with relapsed or refractory multiple myeloma: part 2 of the open-label, multicenter, dose-escalation phase $1 \mathrm{~b}$ study (PAVO). Haematologica. 2020. doi:10.3324/haematol.2019.243790

79. Chari A, San-Miguel J, McCarthy H, et al. Subcutaneous daratumumab plus standard treatment regimens in patients with multiple myeloma across lines of therapy: pleiades study update. Blood. 2019;134 (Supplement_1):3152. doi:10.1182/blood-2019-123560

80. Landgren CO, Chari A, Cohen YC, et al. Daratumumab monotherapy for patients with intermediate-risk or high-risk smoldering multiple myeloma: a randomized, open-label, multicenter, phase 2 study (CENTAURUS). Leukemia. 2020;34(7):1840-1852. doi:10.1038/ s41375-020-0718-z

81. Rajkumar SV, Voorhees PM, Goldschmidt H, et al. Randomized, open-label, phase 3 study of subcutaneous daratumumab (DARA SC) versus active monitoring in patients (Pts) with high-risk smoldering multiple myeloma (SMM): AQUILA. J Clin Oncol. 2018;36 (15_suppl):TPS8062-TPS. doi:10.1200/JCO.2018.36.15_suppl.TPS 8062
Cancer Management and Research

\section{Publish your work in this journal}

Cancer Management and Research is an international, peer-reviewed open access journal focusing on cancer research and the optimal use of preventative and integrated treatment interventions to achieve improved outcomes, enhanced survival and quality of life for the cancer patient.

\section{Dovepress}

The manuscript management system is completely online and includes a very quick and fair peer-review system, which is all easy to use. Visit http://www.dovepress.com/testimonials.php to read real quotes from published authors. 